\title{
Three-Order Multipoint Boundary Value Problems for $p$-Laplacian Operator on Time Scales
}

\author{
Hua Su \\ School of Mathematics and Quantitative Economics, Shandong University of Finance and Economics, Jinan, \\ Shandong 250014, China \\ Correspondence should be addressed to Hua Su; jnsuhua@163.com
}

Received 26 June 2020; Accepted 20 July 2020; Published 14 August 2020

Academic Editor: Liguang Wang

Copyright (c) 2020 Hua Su. This is an open access article distributed under the Creative Commons Attribution License, which permits unrestricted use, distribution, and reproduction in any medium, provided the original work is properly cited.

In this paper, the existence of positive solutions for the nonlinear four-point singular BVP for there-order with $p$-Laplacian operator on time scales will be studied. By using the fixed-point theory, the existence of positive solutions for nonlinear singular boundary value problem with $p$-Laplacian operator on time scales is obtained.

\section{Introduction}

In recent years, the nonlinear boundary value problems have been extensively studied. Recently, for the existence of positive solutions of multipoint boundary value problems, some authors have obtained the existence results. The differential equations offer wonderful tools for describing various natural phenomena arising from natural sciences and engineering, many numerical and analytical results, for example [1-20]. However, the multipoint boundary value problems treated in the above mentioned references do not discuss the problems with singularities and the there-order $p$-Laplacian operator. For the singular case of multipoint boundary value problems for higherorder $p$-Laplacian operator, with the author's acknowledge, no one has studied the existence of positive solutions in this case.

In this paper, we study the following equation with $p$-Laplacian on time scale:

$$
\left(\phi_{p}\left(u^{\Delta \Delta}\right)\right)^{\nabla}+g(t) f\left(u(t), u^{\Delta}(t)\right)=0, \quad 0<t<T
$$

with the following boundary value conditions:

$$
\left\{\begin{array}{l}
u(0)=0, \\
u^{\Delta}(0)-M_{0}\left(u^{\Delta \Delta}(\xi)\right)=0, u^{\Delta}(T)+M_{1}\left(u^{\Delta \Delta}(\eta)\right)=0,
\end{array}\right.
$$

where $\phi_{p}(s)=|s|^{p-2} s, \quad p>1, \phi_{q}=\phi_{p}^{-1},(1 / p)+(1 / q)=1 . \xi, \eta$ $\epsilon(0, T)$ is prescribed and $\xi<\eta, g:(0, T) \rightarrow[0, \infty), M_{0}, M_{1}$ are both nondecreasing continuous odd functions defined on $(-\infty,+\infty)$.

A time scale $\mathbf{T}$ is a nonempty subset and closed subset of R. By an internal $(0, T)$, we always mean the intersection of the real internal $(0, T)$ with the given time scale, that is $(0$, $T) \cap \mathbf{T}$. The operators $\sigma$ and $\rho$ from $\mathbf{T}$ to $\mathbf{T}$ which defined by $[21]$,

$$
\sigma(t)=\inf \{\tau \in \mathbf{T} \mid \tau>t\} \in \mathbf{T}, \rho(t)=\sup \{\tau \in \mathbf{T} \mid \tau<t\} \in \mathbf{T} .
$$

are called the forward jump operator and the backward jump operator, respectively. 
The point $t \in \mathbf{T}$ is left-dense, left-scattered, right-dense, right-scattered if $\rho(t)=t, \rho(t)<t, \sigma(t)=t, \sigma(t)>t$, respectively. If $\mathbf{T}$ has a right scattered minimum $m$, define $\mathbf{T}_{k}=\mathbf{T}-\{m\} ;$ otherwise set $\mathbf{T}_{k}=\mathbf{T}$. If $\mathbf{T}$ has a left scattered maximum $M$, define $\mathbf{T}^{k}=\mathbf{T}-\{M\}$; otherwise set $\mathbf{T}^{k}=\mathbf{T}$.

In this paper, by constructing an integral equation which is equivalent to the problem (1), (2), we research the existence of positive solutions for nonlinear singular boundary value problem (1), (2) when $g$ and $f$ satisfy some suitable conditions.

\section{Preliminaries and Lemmas}

For convenience, in the rest of this article, $\mathbf{T}$ is a closed subset of $\mathbf{R}$ with $0 \in \mathbf{T}_{k}, T \in \mathbf{T}^{k}$.

Letting

$$
B=\left\{u \in C_{l d}[0, T]: u(0)=0\right\} .
$$

Then, $B$ is a Banach space with the norm $\|u\|=\max _{t \in[0, T]}$ $\left|u^{\Delta}(t)\right|$. Suppose

$$
\begin{aligned}
K & =\left\{u \in B: u^{\Delta}(t)\right. \\
& \left.\geq 0, u^{\Delta}(t) \quad \text { is concave function on } t \in[0, T]\right\} .
\end{aligned}
$$

Obviously, $K$ is a cone in $B$ and $0 \leq u^{\Delta}(t) \leq\|u\|$ on $[0, T]$. Set $K_{r}=\{u \in K:\|u\| \leq r\}$.

In the rest of the paper, we make the following assumptions:

$\left(H_{1}\right) f \in C\left([0,+\infty)^{2},[0,+\infty)\right)$;

$\left(H_{2}\right) g \in C_{l d}((0, T),[0,+\infty))$ and there exists $t_{0} \in(0, T)$ which satisfy

$$
g\left(t_{0}\right)>0,0<\int_{0}^{T} g(t) \nabla t<\infty
$$

$\left(H_{3}\right) M_{0}, \quad M_{1} \in((-\infty,+\infty), R)$ are both increasing, continuous, odd functions, and at least one of them satisfies the condition that there exists one $b>0$ which satisfy

$$
0<M_{i}(v) \leq b v, \forall v \geq 0, i=0 \text { or } 1 .
$$

By direct account, From paper [22], we can easy to obtain the following results.

Lemma 1. Suppose condition $\left(\mathrm{H}_{2}\right)$ holds. Then, there exists a constant $L \in(0,(T / 2))$ satisfies

$$
0<\int_{L}^{T-L} g(t) \nabla t<\infty .
$$

Furthermore, the function

$$
\begin{aligned}
A(t)= & \int_{L}^{t} \phi_{q}\left(\int_{s}^{t} g\left(s_{1}\right) \nabla s_{1}\right) \Delta s \\
& +\int_{t}^{T-L} \phi_{q}\left(\int_{t}^{s} g\left(s_{1}\right) \nabla s_{1}\right) \Delta s, \quad t \in[L, T-L],
\end{aligned}
$$

is positive ld-continuous function on $[L, T-L]$, therefore, $A(t)$ has minimum on $[L, T-L]$. Hence, we suppose that there exists constant $B^{\prime}>0$ which satisfy $A \geq B^{\prime}$ on $t \in[L, T-L]$.

Lemma 2. Suppose that conditions $\left(H_{1}\right),\left(H_{2}\right),\left(H_{3}\right)$ hold, $u(t) \in K \cap C^{2}(0, T)$ is a solution of boundary value problems (1), (2) if and only if $u(t) \in B$ is a solution of the following integral equation

$$
u(t)=\int_{0}^{t} w(s) \Delta s,
$$

where

$$
w(t)=\left\{\begin{array}{l}
M_{0} \phi_{q}\left(\int_{\xi}^{\delta} g(s) f\left(u(s), u^{\Delta}(s)\right) \Delta s\right)+\int_{0}^{t} \phi_{q}\left(\int_{s}^{\delta} g(r) f\left(u(r), u^{\Delta}(r)\right) \Delta r\right) \nabla s, 0 \leq \mathrm{t} \leq \delta, \\
M_{1} \phi_{q}\left(\int_{\delta}^{\eta} g(s) f\left(u(s), u^{\Delta}(s)\right) \Delta s\right)+\int_{t}^{T} \phi_{q}\left(\int_{\delta}^{s} g(r) f\left(u(r), u^{\Delta}(r)\right) \Delta r\right) \nabla s, \delta \leq t \leq T .
\end{array}\right.
$$
where

Here, $\delta$ is a unique solution of the equation $g_{1}(t)=g_{2}(t)$,

$$
\begin{aligned}
g_{1}(t)= & M_{0} \phi_{q}\left(\int_{\xi}^{\delta} g(s) f\left(u(s), u^{\Delta}(s)\right) \Delta s\right) \\
& +\int_{0}^{t} \phi_{q}\left(\int_{s}^{\delta} g(r) f\left(u(r), u^{\Delta}(r)\right) \Delta r\right) \nabla s,
\end{aligned}
$$

$$
\begin{aligned}
g_{2}(t)= & M_{1} \phi_{q}\left(\int_{\delta}^{\eta} g(s) f\left(u(s), u^{\Delta}(s)\right) \Delta s\right) \\
& +\int_{t}^{T} \phi_{q}\left(\int_{\delta}^{s} g(r) f\left(u(r), u^{\Delta}(r)\right) \Delta r\right) \nabla s .
\end{aligned}
$$

Equation $g_{1}(t)=g_{2}(t)$ has a unique solution in $(0, T)$. Because $g_{1}(t)$ is strictly monotone increasing on $[0, T)$, and 
$g_{1}(0)=0, g_{2}(t)$ is strictly monotone decreasing on $(0, T]$, and $g_{2}(T)=0$.

Proof. Necessity. By the equation of the boundary condition and $\left(\mathrm{H}_{3}\right)$, we have

$$
u^{\Delta \Delta}(\xi) \geq 0, u^{\Delta \Delta}(\eta) \leq 0
$$

Then, there exist a constant $\delta \in[\xi, \eta] \subset(0, T)$ which satisfy $u^{\Delta \Delta}(\delta)=0$. Firstly, by integrating the equation of the problems $(1)$ on $(\delta, t)$, we have

$$
\phi_{p}\left(u^{\Delta \Delta}(t)\right)=\phi_{p}\left(u^{\Delta \Delta}(\delta)\right)-\int_{\delta}^{t} g(s) f\left(u(s), u^{\Delta}(s)\right) \Delta s,
$$

then

$$
\begin{aligned}
u^{\Delta \Delta}(t) & =-\phi_{q}\left(\int_{\delta}^{t} g(s) f\left(u(s), u^{\Delta}(s)\right) \Delta s\right), \\
u^{\Delta}(t) & =u^{\Delta}(\delta)-\int_{\delta}^{t} \phi_{q}\left(\int_{\delta}^{s} g(r) f\left(u(r), u^{\Delta}(r)\right) \Delta r\right) \nabla s .
\end{aligned}
$$

By $u^{\Delta \Delta}(\delta)=0$ and condition (2), let $t=\eta$ on (15), we have $u^{\Delta \Delta}(1)=-M_{1}\left(u^{\Delta \Delta}(\eta)\right)=M_{1} \phi_{q}\left(\int_{\delta}^{\eta} g(s) f\left(u(s), u^{\Delta}(s)\right) \Delta s\right)$.

Then, we have

$$
\begin{aligned}
u^{\Delta}(\delta)= & M_{1} \phi_{q}\left(\int_{\delta}^{\eta} g(s) f\left(u(s), u^{\Delta}(s)\right) \Delta s\right) \\
& +\int_{\delta}^{T} \phi_{q}\left(\int_{\delta}^{s} g(r) f\left(u(r), u^{\Delta}(r)\right) \Delta r\right) \nabla s .
\end{aligned}
$$

Then

$$
\begin{aligned}
u^{\Delta \Delta}(t)= & M_{1} \phi_{q}\left(\int_{\delta}^{\eta} g(s) f\left(u(s), u^{\Delta}(s)\right) \Delta s\right) \\
& +\int_{t}^{T} \phi_{q}\left(\int_{\delta}^{s} g(r) f\left(u(r), u^{\Delta}(r)\right) \Delta r\right) \nabla s .
\end{aligned}
$$

Therefore, by integrating the above equation (19) on $(0, t)$, we can east to have

$$
\begin{aligned}
u(t)= & \int_{0}^{t} M_{1} \phi_{q}\left(\int_{\delta}^{\eta} g(s) f\left(u(s), u^{\Delta}(s)\right) \Delta s\right) \nabla t \\
& +\int_{0}^{t}\left(\int_{s_{1}}^{T} \phi_{q}\left(\int_{\delta}^{s} g(r) f\left(u(r), u^{\Delta}(r)\right) \Delta r\right) \nabla s\right) \nabla s_{1} .
\end{aligned}
$$

Similarly, for $t \in(0, \delta)$, by integrating the equation of problems $(1)$ on $(0, \delta)$, we have

$$
\begin{aligned}
u(t)= & \int_{0}^{t} M_{0} \phi_{q}\left(\int_{\xi}^{\delta} g(s) f\left(u(s), u^{\Delta}(s)\right) \Delta s\right) \nabla t \\
& +\int_{0}^{t}\left(\int_{s_{1}}^{T} \phi_{q}\left(\int_{s}^{\delta} g(r) f\left(u(r), u^{\Delta}(r)\right) \Delta r\right) \nabla s\right) \nabla s_{1} .
\end{aligned}
$$

Therefore, for any $t \in[0, T], u(t)$ can be expressed as equation $u(t)=\int_{0}^{t} w(s) \Delta s$, where $w(t)$ is expressed as Lemma 3.

Sufficiency. Suppose $u(t)=\int_{0}^{t} w(s) \Delta s$. Then we have

$$
u^{\Delta \Delta}(t)= \begin{cases}\phi_{q}\left(\int_{t}^{\delta} g(s) f\left(u(s), u^{\Delta}(s)\right) \Delta s\right) \geq 0, & 0 \leq t<\delta, \\ -\phi_{q}\left(\int_{\delta}^{t} g(s) f\left(u(s), u^{\Delta}(s)\right) \Delta s\right) \leq 0, & \delta<t \leq T,\end{cases}
$$

So, $\left(\phi_{p}\left(u^{\Delta \Delta}\right)\right)^{\nabla}+g(t) f\left(u(t), u^{\Delta}(t)\right)=0, \quad 0<t<T, \quad t \neq$ $\delta$. These imply that the equation (1) holds. Furthermore, we can easily obtain the boundary value equations of (2). This completes the proof of Lemma 3.

Now, we define an operator $T: K \rightarrow C^{2}[0, T]$ given by

$$
(T u)(t)=\int_{0}^{t} w(s) \Delta s
$$

where $w(t)$ is given by (15). And we can easily obtain the following Lemma.

Lemma 3. Let $u \in K$ and $L$ in Lemma 1. Then

$$
u^{\Delta}(t) \geq L\|u\|, t \in[L, T-L] .
$$

Lemma 4. Suppose that conditions $\left(H_{1}\right),\left(H_{2}\right)$ hold, then for $L \in(0, T / 2)$ in Lemma 1 , we have

$$
u(t) \leq \frac{1}{L} u^{\Delta}(t), t \in[L, T-L]
$$

Proof. If $u(t)$ is the solution of problem (1), (2), then $u^{\Delta}(t)$ is a concave function, and $u(t) \geq 0, u^{\Delta}(t) \geq 0, t \in[0, T]$.

Thus for $t \in[L, T-L]$, we have $u^{\Delta}(t) \geq L\left\|u^{\Delta}\right\|$. Then by $u(t)=\int_{0}^{t} u^{\Delta}(s) \Delta s \leq\left\|u^{\Delta}\right\|$, we have

$$
u(t) \leq \frac{1}{L} u^{\Delta}(t), t \in[L, 1-L]
$$

The proof is complete. 
Remark. : Obviously, we can obtain the following results,

$$
w(0)-M_{0} w^{\prime}(\xi)=0, \quad w(1)+M_{1} w^{\prime}(\eta)=0 .
$$

Furthermore, by Arzela-Ascoli Theorem, it is easy to obtain the following Lemma.

Lemma 5. $T: K \rightarrow K$ is completely continuous.

For convenience, we set

$$
R^{*}=2\left(B^{\prime}\right)^{-1}, R_{*}=\left[(b+1) \phi_{q}\left(\int_{0}^{T} g(r) \Delta r\right)\right]^{-1}
$$

where $B^{\prime}$ and L are given as Lemma 1.

\section{The Existence of Single and Many Positive Solution}

In this section, we present the following five main results.

Theorem 6. Suppose that condition $\left(H_{1}\right),\left(H_{2}\right),\left(H_{3}\right)$ hold. Assume that $f$ also satisfy.

$\left(A_{1}\right)$ For $L r \leq u_{2} \leq r, \quad 0 \leq u_{1} \leq(1 / L) u_{2}$, we have $f\left(u_{1}\right.$, $\left.u_{2}\right) \geq(m r)^{p-1}$;

$\left(A_{2}\right)$ For $0 \leq u_{2} \leq R, 0 \leq u_{1} \leq(1 / L) u_{2}$, we have $f\left(u_{1}\right.$, $\left.u_{2}\right) \leq(M R)^{p-1}$, where $m \in\left(R^{*}, \infty\right), M \in\left(0, R_{*}\right)$. Then, the boundary value problem (1), (2) has at last one solution $u$ such that $\|u\|$ lies between $r$ and $R$.

The proof of Theorem 6. From Condition $\left(H_{3}\right)$, for $\forall v \geq 0$, we can suppose that $0<M_{0}(v) \leq b v$, and $r<R$. By Lemma 3, for any $u \in K$, we can obtain that

$$
u^{\Delta}(t) \geq L\|u\|, t \in[L, T-L]
$$

We define the following two open subset $\Omega_{1}$ and $\Omega_{2}$ of $E$ :

$$
\Omega_{1}=\{u \in K:\|u\|<r\}, \Omega_{2}=\{u \in K:\|u\|<R\} .
$$

For any $u \in \partial \Omega_{1}$, by (29), we have

$$
r=\|u\| \geq u^{\Delta}(t) \geq L\|u\|=L r, t \in[L, T-L] .
$$

For $t \in[L, T-L]$ and $u \in \partial \Omega_{1}$, we shall discuss it from three perspectives.

(i) If $\delta \in[L, T-L]$, thus for $u \in \partial \Omega_{1}$, by $\left(A_{1}\right)$ and Lemma 3 , we have

$$
\begin{aligned}
2(T u)^{\Delta}(\delta) \geq & \int_{0}^{\delta} \phi_{q}\left(\int_{s}^{\delta} g(r) f\left(u(r), u^{\Delta}(r)\right) \nabla r\right) \Delta s \\
& +\int_{\delta}^{T} \phi_{q}\left(\int_{\delta}^{s} g(r) f\left(u(r), u^{\Delta}(r)\right) \nabla r\right) \Delta s \\
\geq & \int_{L}^{\delta} \phi_{q}\left(\int_{s}^{\delta} g(r) f\left(u(r), u^{\Delta}(r)\right) \nabla r\right) \Delta s \\
& +\int_{\delta}^{T-L} \phi_{q}\left(\int_{\delta}^{s} g(r) f\left(u(r), u^{\Delta}(r)\right) \nabla r\right) \Delta s \\
\geq & m r A(\delta) \geq m r B^{\prime}>2 r=2\|u\| .
\end{aligned}
$$

Then, with the case of $\delta \in[L, T-L]$ and $u \in \partial \Omega_{1}$, we have $\|T u\| \geq\|u\|$.

(ii) If $\delta \in(T-L, T]$, thus for $u \in \partial \Omega_{1}$, by $\left(A_{1}\right)$ and Lemma 3, we have

$$
\begin{aligned}
(T u)^{\Delta}(\delta) \geq & M_{0} \phi_{q}\left(\int_{\xi}^{\delta} g(r) f\left(u(r), u^{\Delta}(r)\right) \nabla r\right) \\
& +\int_{0}^{\delta} \phi_{q}\left(\int_{s}^{\delta} g(r) f\left(u(r), u^{\Delta}(r)\right) \nabla r\right) \Delta s \\
\geq & \int_{L}^{T-L} \phi_{q}\left(\int_{s}^{T-L} g(r) f\left(u(r), u^{\Delta}(r)\right) \nabla r\right) \Delta s \\
\geq & m r A(1-L) \geq m r B^{\prime}>2 r>r=\|u\| .
\end{aligned}
$$

Then, with the case of $\delta \in(T-L, T]$ and $u \in \partial \Omega_{1}$, we have $\|T u\| \geq\|u\|$.

(iii) If $\delta \in(0, L)$, thus for $u \in \partial \Omega_{1}$, by $\left(A_{1}\right)$ and Lemma 3, we have

$$
\begin{aligned}
(T u)^{\Delta}(\delta) \geq & M_{1} \phi_{q}\left(\int_{\delta}^{\eta} g(r) f\left(u(r), u^{\Delta}(r)\right) \nabla r\right) \\
& +\int_{\delta}^{1} \phi_{q}\left(\int_{\delta}^{s} g(r) f\left(u(r), u^{\Delta}(r)\right) \nabla r\right) \Delta s \\
\geq & \int_{L}^{T-L} \phi_{q}\left(\int_{L}^{s} g(r) f\left(u(r), u^{\Delta}(r)\right) \nabla r\right) \Delta s \\
\geq & m r A(L) \geq m r B^{\prime}>2 r>r=\|u\| .
\end{aligned}
$$

Then, with the case of $\delta \in(0, L)$ and $u \in \partial \Omega_{1}$, we have $\|T u\| \geq\|u\|$.

Therefore, for any case of $\delta \in(0, T-L]$, we all easy to obtain that

$$
\|T u\|>\|u\|, \forall u \in \partial \Omega_{1}
$$

Then, by fixed point theorem of cone expansioncompression type in $[23,24]$, we have

$$
i\left(T, \Omega_{1}, K\right)=0
$$


Secondly, for $u \in \partial \Omega_{2}$, using $u^{\Delta}(t) \leq\|u\|=R$, from $\left(A_{2}\right)$, we can easily know that

$$
\begin{aligned}
(T u)^{\Delta}(\delta) \leq & M_{0} \phi_{q}\left(\int_{0}^{T} g(r) f\left(u(r), u^{\Delta}(r)\right) \Delta r\right) \\
& +\int_{0}^{T} \phi_{q}\left(\int_{s}^{\delta} g(r) f\left(u(r), u^{\Delta}(r)\right) \nabla r\right) \Delta s \\
\leq & b M R \phi_{q}\left(\int_{0}^{T} g(r) \Delta r\right)+M R \phi_{q}\left(\int_{0}^{T} g(r) \Delta r\right) \\
= & (b+1) M R \phi_{q}\left(\int_{0}^{T} g(r) \Delta r\right) \leq R=\|u\| .
\end{aligned}
$$

Thus, we have

$$
\|T u\|<\|u\|, \forall u \in \partial \Omega_{2} .
$$

Then, by fixed point theorem of cone expansioncompression type in $[23,24]$, we have

$$
i\left(T, \Omega_{2}, K\right)=1 \text {. }
$$

Therefore, by (36), (39), $r<R$ we have

$$
i\left(T, \Omega_{2} \backslash \bar{\Omega}_{1}, K\right)=1 .
$$

Then, operator $T$ has at last one fixed point $u \in\left(\Omega_{2} \backslash \bar{\Omega}_{1}\right)$, and $r \leq\|u\| \leq R$. This completes the proof of Theorem 6 .

Theorem 7. Suppose that condition $\left(H_{1}\right),\left(H_{2}\right),\left(H_{3}\right)$ hold. Assume that $f$ also satisfy.

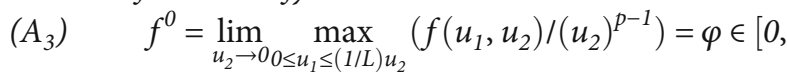

$$
\begin{aligned}
& \left.\left(R_{*} / 4\right)^{p-1}\right) \text {; } \\
& \left(A_{4}\right) \quad f_{\infty}=\lim _{u_{2} \rightarrow \infty} \min _{0 \leq u_{1} \leq(1 / L) u_{2}}\left(f\left(u_{1}, u_{2}\right) /\left(u_{2}\right)^{p-1}\right)=\psi \in((2 \\
& \left.R^{*}(L)^{p-1}, \infty\right)
\end{aligned}
$$

Then, the boundary value problem (1), (2) has at last one solution $u$.

The proof of Theorem 7 .

First, by $0 \leq \lim _{u_{2} \rightarrow 0 \leq u_{1} \leq(1 / L) u_{2}}\left(f\left(u_{1}, u_{2}\right) /\left(u_{2}\right)^{p-1}\right)=\varphi<$ $\left(R_{*} / 4\right)^{p-1}$, letting $\varepsilon=\left(R_{*} / 4\right)^{p-1}-\varphi>0$, we know that there exists an appropriately small positive number $\rho$ which satisfy as $0 \leq u_{2} \leq \rho, u_{2} \neq 0$, and we have

$$
f\left(u_{1}, u_{2}\right) \leq(\varphi+\varepsilon)\left(u_{2}\right)^{p-1} \leq\left(R_{*} / 4\right)^{p-1} \rho^{p-1}=\left(\left(R_{*} / 4\right) \rho\right)^{p-1} .
$$

Then, letting $R=\rho, 0<M=R_{*} / 4<R_{*}$, thus by the above equation, we can have

$$
f\left(u_{1}, u_{2}\right) \leq(M R)^{p-1}, 0 \leq u_{2} \leq R, 0 \leq u_{1} \leq \frac{1}{L} u_{2} .
$$

So condition $\left(A_{2}\right)$ in Theorem 6 holds.

Next, by $\left(2 R^{*} / L\right)^{p-1}<\lim _{u_{2} \rightarrow \infty} \min _{0 \leq u_{1} \leq(1 / L) u_{2}}\left(f\left(u_{1}, u_{2}\right) /\left(u_{2}\right)^{p-1}\right)$ $=\psi<\infty$, letting $\varepsilon=\psi-\left(2 R^{*} / L\right)^{p-1}$, we know that there exists an adequately big positive number $r \neq R$ which satisfy as $u_{2} \geq L r, \quad 0 \leq u_{1} \leq(1 / L) u_{2}$, and we have

$$
f\left(u_{1}, u_{2}\right) \geq(\psi-\varepsilon)\left(u_{2}\right)^{p-1} \geq\left(\frac{2 R^{*}}{L}\right)^{p-1}(L r)^{p-1}=\left(2 R^{*} r\right)^{p-1},
$$

Letting $m=2 R^{*}>R^{*}$, thus by the above equation, we have that $\left(A_{1}\right)$ in Theorem 6 holds. Therefore, by Theorem 6 , we can easily obtain the results of Theorem 7 holds. The proof of Theorem 3.2 is complete.

Corollary 8. Suppose that condition $\left(\mathrm{H}_{1}\right),\left(\mathrm{H}_{2}\right),\left(\mathrm{H}_{3}\right)$ hold. Assume that $f$ also satisfy.

$$
\left(A_{5}\right) \quad f^{\infty}=\lim _{u_{2} \rightarrow \infty} \max _{0 \leq u_{1} \leq(1 / L) u_{2}}\left(f\left(u_{1}, u_{2}\right) /\left(u_{2}\right)^{p-1}\right)=\lambda \in[0,
$$
$\left.\left(R_{*} / 4\right)^{p-1}\right)$;

$$
\text { (A } \left.f_{6}\right) f_{0}=\lim _{u_{2} \rightarrow 00 \leq u_{1} \leq(1 / L) u_{2}}\left(f\left(u_{1}, u_{2}\right) /\left(u_{2}\right)^{p-1}\right)=\varphi \in\left(\left(2 R^{*} /\right.\right.
$$
$\left.L)^{p-1}, \infty\right)$.

Then, the boundary value problem (1), (2) has at last one solution $u$.

The proof of Corollary 8. Similar to the proof of Theorem 7 , we can obtain Corollary 8 .

Theorem 9. Suppose that conditions $\left(\mathrm{H}_{1}\right),\left(\mathrm{H}_{2}\right),\left(\mathrm{H}_{3}\right)$, and $\left(A_{2}\right)$ in Theorem 6 hold. Assume that $f$ also satisfy.

$$
\begin{aligned}
& \left.\left(A_{7}\right) f_{0}=\lim _{u_{2} \rightarrow 00 \leq u_{1} \leq(1 / L) u_{2}} \min _{\left(A_{8}\right) f_{\infty}=\lim _{u_{2} \rightarrow \infty} \min _{0 \leq u_{1} \leq(1 / L) u_{2}}\left(f\left(u_{1}, u_{2}\right) /\left(u_{2}\right) /\left(u_{2}\right)^{p-1}\right)=+\infty ;}\right)^{p-1}=+\infty .
\end{aligned}
$$

Then, the boundary value problem (1), (2) have at least two solutions $u_{1}, u_{2}$ which satisfy

$$
0<\left\|u_{1}\right\|<R<\left\|u_{2}\right\| .
$$

The proof of Theorem 9.

Firstly, by $\lim _{u_{2} \rightarrow 0} \min _{0 \leq u_{1} \leq(1 / L) u_{2}}\left(f\left(u_{1}, u_{2}\right) /\left(u_{2}\right)^{p-1}\right)=+\infty$, for any $M>\left(2 / B^{\prime} L\right)$, there exists a constant $\rho_{*} \in(0, R)$ which satisfy

$$
f\left(u_{1}, u_{2}\right) \geq\left(M u_{2}\right)^{p-1}, 0<u_{2} \leq \rho_{*}, 0 \leq u_{1} \leq \frac{1}{L} u_{2} .
$$

Set $\Omega_{\rho_{*}}=\left\{u \in K:\|u\|<\rho_{*}\right\}$, similar to the previous proof of Theorem 6 , for any $u \in \partial \Omega_{\rho_{*}}$, from the above 
discussion and Lemma 2, we can have from three perspectives

$$
\|T u\| \geq\|u\|, \forall u \in \partial \Omega_{\rho_{*}}
$$

Then, by fixed point theorem of cone expansioncompression type, we can have

$$
i\left(T, \Omega_{\rho_{*}}, K\right)=0
$$

Secondly, for any $\bar{M}>\left(2 / B^{\prime} L\right)$, by $\lim _{u_{2} \rightarrow \infty} \min _{0 \leq u_{1} \leq(1 / L) u_{2}}\left(f\left(u_{1}\right.\right.$, $\left.\left.u_{2}\right) /\left(u_{2}\right)^{p-1}\right)=+\infty$, there exists a constant $\rho_{0}>0$ which satisfy

$$
f\left(u_{1}, u_{2}\right) \geq\left(\bar{M} u_{2}\right)^{p-1}, u_{2}>\rho_{0}, 0 \leq u_{1} \leq \frac{1}{L} u_{2}
$$

Therefore, we choose a constant $\rho^{*}>\max \left\{R, \rho_{0} / L\right\}$, obviously $\rho_{*}<R<\rho^{*}$. Set $\Omega_{\rho^{*}}=\left\{u \in K:\|u\|<\rho^{*}\right\}$. For any $u \in$ $\partial \Omega_{\rho^{*}}$, by Lemma 2 , we can easily obtain

$$
u^{\Delta}(t) \geq L\|u\|=L \rho^{*}>\rho_{0}, t \in[L, T-L]
$$

Then, by the above discussion and also similar to the previous proof of Theorem 6, we can also have from three perspectives

$$
\|T u\| \geq\|u\|, \forall u \in \partial \Omega_{\rho^{*}}
$$

Then, by fixed point theorem of cone expansioncompression type, we have

$$
i\left(T, \Omega_{\rho^{*}}, K\right)=0
$$

Finally, imitating the latter proof of Theorem 6 , for any $u$ $\in \partial \Omega_{R}$, by $\left(A_{2}\right)$, setting $\Omega_{R}=\{u \in K:\|u\|<R\}$, we can also easy to have

$$
\|T u\| \leq\|u\|, \forall u \in \partial \Omega_{R}
$$

Then, by fixed point theorem of cone expansioncompression type, we have

$$
i\left(T, \Omega_{R}, K\right)=1
$$

Therefore, by (47), (51), (53), $\rho_{*}<R<\rho^{*}$ we have

$$
i\left(T, \Omega_{R} \backslash \bar{\Omega}_{\rho_{*}}, K\right)=1, i\left(T, \Omega_{\rho^{*}} \backslash \bar{\Omega}_{R}, K\right)=-1 .
$$

Then, $T$ have fixed point $u_{1} \in \Omega_{R} \backslash \bar{\Omega}_{\rho_{*}}$, and fixed point $u_{2} \in \Omega_{\rho^{*}} \backslash \bar{\Omega}_{R}$.

Obviously, $u_{1}, u_{2}$ are all positive solutions of problem (1), (2) and $0<\left\|u_{1}\right\|<R<\left\|u_{2}\right\|$. The proof of Theorem 9 is complete.

Theorem 10. Suppose that conditions $\left(H_{1}\right),\left(H_{2}\right),\left(H_{3}\right)$ and $\left(A_{1}\right)$ in Theorem 6 hold. Assume that $f$ also satisfy.

$$
\begin{aligned}
& \left(A_{9}\right) f^{0}=\lim _{u_{2} \rightarrow 0 \leq u_{1} \leq(1 / L) u_{2}} \max _{1}\left(f\left(u_{1}, u_{2}\right) /\left(u_{2}\right)^{p-1}\right)=0 ; \\
& \left(A_{10}\right) f^{\infty}=\lim _{u_{2} \rightarrow \infty} \max _{0 \leq u_{1} \leq(1 / L) u_{2}}\left(f\left(u_{1}, u_{2}\right) /\left(u_{2}\right)^{p-1}\right)=0 .
\end{aligned}
$$

Then, the boundary value problem (1), (2) have at least two solutions $u_{1}, u_{2}$ which satisfy $0<\left\|u_{1}\right\|<r<\left\|u_{2}\right\|$.

The proof of Theorem 10.

Firstly, by $\lim _{u_{2} \rightarrow \infty} \max _{0 \leq u_{1} \leq(1 / L) u_{2}}\left(f\left(u_{1}, u_{2}\right) /\left(u_{2}\right)^{p-1}\right)=0$, for $\eta_{1}$ $\in\left(0, R_{*}\right)$, there exists a constant $\rho_{*} \in(0, r)$ which satisfy

$$
f\left(u_{1}, u_{2}\right) \leq\left(\eta_{1} u_{2}\right)^{p-1}, 0<u_{2} \leq \rho_{*}, 0 \leq u_{1} \leq \frac{1}{L} u_{2} .
$$

Set $\Omega_{\rho_{*}}=\left\{u \in K:\|u\|<\rho_{*}\right\}$, for any $u \in \partial \Omega_{\rho_{*}}$, by (23), we have

$$
\begin{aligned}
\|T u\|= & (T u)^{(n-2)}(\delta) \leq M_{0} \phi_{q}\left(\int_{0}^{T} g(r) f\left(u(r), u^{\Delta}(r)\right) \nabla r\right) \\
& +\int_{0}^{T} \phi_{q}\left(\int_{s}^{\delta} g(r) f\left(u(r), u^{\Delta}(r)\right) \nabla r\right) \Delta s \\
\leq & M_{0} \phi_{q}\left(\int_{0}^{T} g(r) f\left(u(r), u^{\Delta}(r)\right) \nabla r\right) \\
& +\phi_{q}\left(\int_{0}^{T} g(r) f\left(u(r), u^{\Delta}(r)\right) \nabla r\right) \\
\leq & (b+1) \eta_{1} \rho_{*} \phi_{q}\left(\int_{0}^{T} g(r) d r\right) \leq \rho_{*}=\|u\| .
\end{aligned}
$$

Then, by fixed point theorem of cone expansioncompression type, we have

$$
i\left(T, \Omega_{\rho_{*}}, K\right)=1
$$

Secondly, letting $f^{*}(x)=\max _{0 \leq u_{n-1} \leq x, 0 \leq u_{1} \leq(1 / L) u_{2}} f\left(u_{1}, u_{2}\right)$, we can easy to know that $f^{*}(x)$ is monotone increasing with respect to $x \geq 0$.

Therefore by $\lim _{u_{2} \rightarrow \infty} \max _{0 \leq u_{1} \leq(1 / L) u_{2}}\left(f\left(u_{1}, u_{2}\right) /\left(u_{2}\right)^{p-1}\right)=0$, we can easy to have $\lim _{x \rightarrow \infty}\left(f^{*}(x) / x^{p-1}\right)=0$.

Therefore, for any $\eta_{2} \in\left(0, R_{*}\right)$, there exists a constant $\rho^{*}>r$ which satisfy

$$
f^{*}(x) \leq\left(\eta_{2} x\right)^{p-1}, x \geq \rho^{*}
$$

Set $\Omega_{\rho^{*}}=\left\{u \in K:\|u\|<\rho^{*}\right\}$, for any $u \in \partial \Omega_{\rho^{*}}$, by (4.8), we have 


$$
\begin{aligned}
\|T u\|= & (T u)^{\Delta}(\delta) \leq M_{0} \phi_{q}\left(\int_{0}^{T} g(r) f\left(u(r), u^{\Delta}(r)\right) \nabla r\right) \\
& +\int_{0}^{T} \phi_{q}\left(\int_{s}^{\delta} g(r) f\left(u(r), u^{\Delta}(r)\right) \nabla r\right) \Delta s \\
\leq & M_{0} \phi_{q}\left(\int_{0}^{T} g(r) f\left(u(r), u^{\Delta}(r)\right) \nabla r\right) \\
& +\phi_{q}\left(\int_{0}^{T} g(r) f\left(u(r), u^{\Delta}(r)\right) \nabla r\right) \\
\leq & (b+1) \phi_{q}\left(\int_{0}^{T} g(r) f^{*}\left(\rho^{*}\right) d r\right) \\
\leq & (b+1) \eta_{2} \rho^{*} \phi_{q}\left(\int_{0}^{T} g(r) d r\right) \leq \rho^{*}=\|u\| .
\end{aligned}
$$

Then, by fixed point theorem of cone expansioncompression type, we have

$$
i\left(T, \Omega_{\rho^{*}}, K\right)=1 .
$$

Finally, imitating the previous proof of Theorem 6, for any $u \in \partial \Omega_{r}$, by $\left(A_{1}\right)$, setting $\Omega_{r}=\{u \in K:\|u\|<r\}$, For any $u \in \partial \Omega_{r}$, we can also easy to have

$$
\|T u\| \geq\|u\|, \forall u \in \partial \Omega_{r} .
$$

Then, by fixed point theorem of cone expansioncompression type, we have

$$
i\left(T, \Omega_{r}, K\right)=0 .
$$

Therefore, by (57), (60), (62), $\rho_{*}<r<\rho^{*}$, we have

$$
i\left(T, \Omega_{r} \backslash \bar{\Omega}_{\rho_{*}}, K\right)=-1, i\left(T, \Omega_{\rho^{*}} \backslash \bar{\Omega}_{r}, K\right)=1 .
$$

Then, $T$ have fixed point $u_{1} \in \Omega_{r} \backslash \bar{\Omega}_{\rho_{*}}$, and fixed point $u_{2} \in \Omega_{\rho_{x}} \backslash \bar{\Omega}_{r}$.

Obviously, $u_{1}, u_{2}$ are all positive solutions of problem (1),(2) and $0<\left\|u_{1}\right\|<r<\left\|u_{2}\right\|$. The proof of Theorem 10 is complete.

\section{Application}

Example. Consider the following three-order BVP with $p$-Laplacian

$$
\left\{\begin{array}{l}
\left(\phi_{p}\left(u^{\prime \prime}\right)\right)^{\prime}+\frac{1}{64 \pi^{4}} t^{-\frac{1}{2}}(1-t)\left[\ln u^{5}+e^{\left(u^{\prime}\right)^{2}}\right]=0,0<t<1, \\
u(0)=0 \\
u^{\prime}(0)-u^{\prime \prime}(0.25)=0, u^{\prime}(1)+5 u^{\prime \prime}(0.3)=0,
\end{array}\right.
$$

where $p=4, \xi=0.25, \eta=0.3, \quad B^{\prime}=1 / 4$,

$$
g(t)=\frac{1}{64 \pi^{4}} t^{-\frac{1}{2}}(1-t), f\left(u_{1}, u_{2}\right)=\ln u^{5}+e^{\left(u^{\prime}\right)^{2}} .
$$

Then obviously, $q=4 / 3, \int_{0}^{1} g(t) d t=1 / 64 \pi^{3}, f_{\infty}=+\infty$, $f_{0}=+\infty$,

$$
M_{0}(v)=v<2 v=b v, M_{1}(v)=5 v, \forall v \geq 0,
$$

so conditions $\left(H_{1}\right),\left(H_{2}\right),\left(H_{3}\right),\left(A_{7}\right),\left(A_{8}\right)$ hold.

Next, $\phi_{q}\left(\int_{0}^{1} g(t) d t\right)=1 / 4 \pi, R_{*}=4 \pi / 3$, we choose $R=3$, $M=2$ and for $B^{\prime}=1 / 4$, because of the monotone increasing of $f\left(u_{1}, u_{2}\right)$ on $[0, \infty) \times[0, \infty)$, then

$$
f\left(u_{1}, \quad u_{2}\right) \leq f(12,3)=286, \quad 0 \leq u_{2} \leq 3, \quad 0 \leq u_{1} \leq 4 u_{2} .
$$
know

Therefore, using $0<M<R_{*}$, we have $(M R)^{p-1}=328$, we

$$
f\left(u_{1}, \quad u_{2}\right) \leq(M R)^{p-1}, \quad 0 \leq u_{2} \leq 3, \quad 0 \leq u_{1} \leq 4 u_{2},
$$

so conditions $\left(A_{2}\right)$ holds. Then, by Theorem 9, the Example has at least two positive solutions $v_{1}, \quad v_{2}$ and $0<\left\|v_{1}\right\|<3<\|$ $v_{2} \|$.

\section{Data Availability}

No data were used to support this study.

\section{Conflicts of Interest}

The authors declare that there is no conflict of interest regarding the publication of this paper.

\section{Authors' Contributions}

The study was carried out in collaboration among all authors. All authors read and approved the final manuscript.

\section{Acknowledgments}

The authors really appreciate the anonymous reviewers for their pertinent comments and suggestions, which were helpful to improve the earlier manuscript. The author was supported by the Project of National Social Science Fund of China (NSSF) (18BTY015) and Shandong Province Higher Educational Science and Technology Program (J16LI01).

\section{References}

[1] X. Liu, M. Jia, and X. Xiang, "On the solvability of a fractional differential equation model involving the $p$-Laplacian operator," Computers and Mathematics with Applications, vol. 64, no. 10, pp. 3267-3275, 2012.

[2] X. Yang, "Green's function and positive solutions for higherorder ODE," Applied Mathematics and Computation, vol. 136, no. 2-3, pp. 379-393, 2003. 
[3] F. Atici and G. S. Guseinov, "On Green's functions and positive solutions for boundary value problems on time scales," Journal of Computational and Applied Mathematics, vol. 141, no. 1-2, pp. 75-99, 2002.

[4] X. Zhang, Z. Shao, and Q. Zhong, "Positive solutions for semipositone $(k, n-k)$ conjugate boundary value problems with singularities on space variables," Applied Mathematics Letters, vol. 72, pp. 50-57, 2017.

[5] X. Zhang, L. Liu, and Y. Wu, "The entire large solutions for a quasilinear Schrödinger elliptic equation by the dual approach," Applied Mathematics Letters, vol. 55, pp. 1-9, 2016.

[6] R. Liang, J. Peng, and J. Shen, "Double positive solutions for a nonlinear four-point boundary value problem with a $p$ -Laplacian operator," Nonlinear Analysis: Theory, Methods \& Applications, vol. 68, no. 7, pp. 1881-1889, 2008.

[7] E. R. Kaufmann, "Positive solutions of a three-point boundary value problem on a time scale," Electronic Journal of Differential Equations (EJDE), vol. 82, pp. 1-11, 2003.

[8] X. Hao, P. Zhang, and L. Liu, "Iterative solutions of singular $(k, n-k)$ conjugate boundary value problems with dependence on the derivatives," Applied Mathematics Letters, vol. 27, pp. 64-69, 2014.

[9] X. Zhang, L. Liu, Y. Wu, and Y. Cui, "Existence of infinitely solutions for a modified nonlinear Schrödinger equation via dual approach," Electronic Journal of Differential Equations, vol. 2147, pp. 1-15, 2018.

[10] J. He, X. Zhang, L. Liu, and Y. Wu, "Existence and nonexistence of radial solutions of the Dirichlet problem for a class of general k-hessian equations," Nonlinear Analysis: Modelling and Control, vol. 23, no. 4, pp. 475-492, 2018.

[11] X. Zhang, Y. Wu, and Y. Cui, "Existence and nonexistence of blow-up solutions for a Schrödinger equation involving a nonlinear operator," Applied Mathematics Letters, vol. 82, pp. 8591, 2018

[12] J. He, X. Zhang, L. Liu, Y. Wu, and Y. Cui, "Existence and asymptotic analysis of positive solutions for a singular fractional differential equation with nonlocal boundary conditions," Boundary Value Problems, vol. 2018, no. 1, 2018.

[13] X. Zhang, L. Liu, Y. Wu, and Y. Cui, "New result on the critical exponent for solution of an ordinary fractional differential problem," Journal of Function Spaces, vol. 2017, Article ID 3976469, 4 pages, 2017.

[14] X. Zhang, L. Liu, Y. Wu, and Y. Cui, "The existence and nonexistence of entire large solutions for a quasilinear Schrödinger elliptic system by dual approach," Journal of Mathematical Analysis and Applications, vol. 464, no. 2, pp. 1089-1106, 2018.

[15] C. Chen, K. Li, Y. Chen, and Y. Huang, "Two-grid finite element methods combined with Crank-Nicolson scheme for nonlinear Sobolev equations," Advances in Computational Mathematics, vol. 45, no. 2, pp. 611-630, 2019.

[16] X. Zhang, J. Xu, J. Jiang, Y. Wu, and Y. Cui, “The convergence analysis and uniqueness of blow-up solutions for a Dirichlet problem of the general $k$-Hessian equations," Applied Mathematics Letters, vol. 102, article 106124, 2020.

[17] X. Liu, M. Jia, and W. Ge, "The method of lower and upper solutions for mixed fractional four-point boundary value problem with $p$-Laplacian operator," Applied Mathematics Letters, vol. 65, pp. 56-62, 2017.

[18] H. Zhang, Y. Li, and J. Xu, "Positive solutions for a system of fractional integral boundary value problems involving
Hadamard-type fractional derivatives," Complexity, vol. 2019, Article ID 2671539, 11 pages, 2019.

[19] T. Qi, Y. Liu, and Y. Cui, "Existence of solutions for a class of coupled fractional differential systems with nonlocal boundary conditions," Journal of Function Spaces, vol. 2017, Article ID 6703860, 9 pages, 2017.

[20] B. Liu and Y. Liu, "Positive solutions of a two-point boundary value problem for singular fractional differential equations in Banach space," Journal of Function Spaces and Applications, vol. 2013, article 585639, 9 pages, 2013.

[21] S. Hilger, "Analysis on measure chains - a unified approach to continuous and discrete calculus," Results in Mathematics, vol. 18, no. 1-2, pp. 18-56, 1990.

[22] H. R. Sun and W. T. Li, "Positive solutions for nonlinear threepoint boundary value problems on time scales," Journal of Mathematical Analysis and Applications, vol. 299, no. 2, pp. 508-524, 2004.

[23] D. Guo and V. Lakshmikantham, Nonlinear Problems in Abstract Cone, Academic Press, Sandiego, 1988.

[24] D. Guo, V. Lakshmikantham, and X. Liu, Nonlinear Integral Equations in Abstract Spaces, Kluwer Academic Publishers, 1996. 